\title{
Translation of Collagen Ultrastructure to Biomaterial Fabrication for Material-Independent but Highly Efficient Topographic Immunomodulation
}

\author{
Matthias Ryma, Tina Tylek, Julia Liebscher, Carina Blum, Robin Fernandez, \\ Christoph Böhm, Wolfgang Kastenmüller, Georg Gasteiger, and Jürgen Groll**
}

Supplement-free induction of cellular differentiation and polarization solely through the topography of materials is an auspicious strategy but has so far significantly lagged behind the efficiency and intensity of media-supplementation-based protocols. Consistent with the idea that 3D structural motifs in the extracellular matrix possess immunomodulatory capacity as part of the natural healing process, it is found in this study that human-monocyte-derived macrophages show a strong $\mathrm{M} 2 \mathrm{a}-$-like prohealing polarization when cultured on type I rat-tail collagen fibers but not on collagen I films. Therefore, it is hypothesized that highly aligned nanofibrils also of synthetic polymers, if packed into larger bundles in 3D topographical biomimetic similarity to native collagen I, would induce a localized macrophage polarization. For the automated fabrication of such bundles in a 3D printing manner, the strategy of "melt electrofibrillation" is pioneered by the integration of flow-directed polymer phase separation into melt electrowriting and subsequent selective dissolution of the matrix polymer postprocessing. This process yields nanofiber bundles with a remarkable structural similarity to native collagen I fibers, particularly for medical-grade poly( $\varepsilon$-caprolactone). These biomimetic fibrillar structures indeed induce a pronounced elongation of human-monocyte-derived macrophages and unprecedentedly trigger their M2-like polarization similar in efficacy as interleukin-4 treatment.

\section{Introduction}

In vivo, cells are embedded in an organ and tissue-specific 3D environment (also called extracellular matrix (ECM)) that comprises a mixture of soluble bioactive molecules such as growth factors and cytokines, as well as a variety of 3D physical cues from the nano- to the microscale. ${ }^{[1]}$ For a long time, the structural component was merely seen as a scaffold for the cells, while cellular behavior and development were assumed to mainly be orchestrated by the soluble factors and paracrine signaling. It has, however, become clear that the role of the insoluble matrix component is much richer than simply serving as a substrate for cell adhesion. Aside from the importance of mechanical properties, 3D structural motifs, as pore size and shape or fiber alignment, are meanwhile known to possess cell regulatory functions beyond mere adhesion in physiological ${ }^{[2]}$ and also pathological ${ }^{[3]}$ situations.

This opened a promising strategy to influence or even direct cellular behavior

M. Ryma, Dr. T. Tylek, Dr. J. Liebscher, Dr. C. Blum, Dr. R. Fernandez, C. Böhm, Prof. J. Groll

Department of Functional Materials in Medicine and Dentistry at the Institute of Functional Materials and Biofabrication

and Bavarian Polymer Institute

Julius-Maximilians-Universität Würzburg

97070 Würzburg, Germany

E-mail: juergen.groll@fmz.uni-wuerzburg.de

Prof. W. Kastenmüller, Prof. G. Gasteiger

Würzburg Institute of Systems Immunology

Max Planck Research Group at the Julius-Maximilians-Universität

Würzburg

97080 Würzburg, Germany

The ORCID identification number(s) for the author(s) of this article can be found under https://doi.org/10.1002/adma.202101228.

(C) 2021 The Authors. Advanced Materials published by Wiley-VCH $\mathrm{GmbH}$. This is an open access article under the terms of the Creative Commons Attribution-NonCommercial-NoDerivs License, which permits use and distribution in any medium, provided the original work is properly cited, the use is non-commercial and no modifications or adaptations are made.

DOI: 10.1002/adma.202101228 by controlling the material topography in a complementary way to more established approaches such as the release of bioactive molecules ${ }^{[4]}$ or the biofunctionalization of material interfaces. ${ }^{[5]}$ For example, it has been shown that the topography, e.g., substrate roughness, grooves, and aligned or random fiber surfaces, can influence cell geometry/morphology and functions such as adhesion, ${ }^{[6]}$ migration, ${ }^{[7]}$ and differentiation. ${ }^{[8]}$

In this context, the manipulation of cells of the innate immune system through controlled material topography in order to improve healing capacity in regenerative medicine approaches and reduce rejection of implants in situ ${ }^{[4]}$ has for far been underappreciated. Especially, macrophages as key mediators between the implant and the body are a crucial target. ${ }^{[9]}$ As highly plastic cells, macrophage phenotypes represent a spectrum of polarization and activation. ${ }^{[10]}$ However, based on the endpoints of this spectrum, macrophages can be roughly classified into two main subgroups: the "classically activated" proinflammatory M1 type and the "alternatively activated" antiinflammatory or prohealing M2 type. Nevertheless, they still 
can switch their polarization state depending on the environment and additional stimuli. ${ }^{[11]}$ Reported attempts to influence macrophage polarization through controlled material topographies and geometries comprise aligned topographies in $2 \mathrm{D},{ }^{[12]}$ hydrogels with highly regular spherical pores, ${ }^{[13]}$ and microfiber-based scaffolds. ${ }^{[14]}$ All these approaches have, however, so far focused on nonbiomimetic material designs, resulting in nonphysiological stimulation of the macrophages and intensity and efficiency of the influence on macrophage polarization that is far below the standard induction protocols by media supplements such as Interleukin (IL)-4 or dexamethasone (DEX). ${ }^{[15]}$

Interestingly, the influence of ECM physical properties on macrophage phenotype and behavior has recently become an active field of research. Physical factors, such as tension and the matrix's mechanical properties, were indicated to play a role in macrophage function and phenotype. ${ }^{[16]}$ Furthermore, physical alterations in the architecture of the $\mathrm{ECM}^{[17]}$ which majorly consists of collagen fibers and fibrils, were assumed to impact macrophage polarization. ${ }^{[12]}$ However, studies that clearly demonstrate the relevance of ECM structure for macrophage polarization are missing. Collagen forms highly organized, aligned structured 3D scaffolds around the cells and are further known to be involved in every phase of wound healing. ${ }^{[18]}$ The core structural element is nanofibrils that are organized in bundles of micrometer-sized diameter. These highly organized and anisotropic structural elements have so far not been examined regarding their potential importance for macrophage behavior. We hypothesized that the fibrillar structure of collagen is a central factor in influencing the macrophage response and examined the reaction of primary human-monocyte-derived macrophages to collagen fibers and fibrils. These studies revealed that collagen type I fibrils of decellularized rat tail tendon led to an M2-like polarization, with upregulation of M2 markers and downregulation of M1 markers over time, of human macrophages in correlation with an elongated morphology, leading to a similar expression pattern to the standard biochemical stimulation (Figure S1, Supporting Information). This observation delivered first evidence for the biological importance of ECM-component induced cell morphological changes for the polarization of human macrophages. It furthermore gave rise to the hypothesis that the hierarchical 3D structural organization of the collagen fibers, and not the biochemical properties of the protein itself, acts as the main trigger for macrophage elongation and thus for the induction of polarization. This opened a new biomimetic design criterium and strategy for topography-based immunomodulatory material design as the basis for our subsequent studies.

\section{Melt Electrofibrillation}

To verify this hypothesis, we first had to develop a fabrication method for collagen-mimetic nanofibril bundles. We hypothesized that using polymer blends instead of homopolymers for melt electrowriting (MEW), ${ }^{[19]}$ oriented phase separation, and thus a fibrillary internal structure of the printed filament can be achieved. Selective dissolution of the higher volume share polymer (matrix polymer) would then result in remaining biomimetic nanofibrillar microbundles. We termed this concept melt electrofibrillation (Figure 1a). We developed this method with poly(2-oxazoline)s, as they are a polymer family that gained increased interest in the recent years ${ }^{[20]}$ based mainly on their biocompatibility, ${ }^{[21]}$ thermoresponsive behavior, ${ }^{[22]}$ and several applications in pharmacy ${ }^{[23]}$ and biofabrication. ${ }^{[24]}$ In particular, amorphous poly(2-cyclopropyl-2-oxazoline) (PcycloPrOx) can be dissolved in water by temperature decrease below its LCST and can therefore act as matrix polymer, while poly(2-n-propyl-2-oxazoline) (PnPrOx) and poly(2-isopropyl-2-oxazoline) (PiPrOx) lose their dissolution-behavior by temperature dependent crystallization (Figures S10 and S15, Supporting Information). At first, homogenous fibers of PnPrOx in diameters ranging from 60 to up to $170 \mu \mathrm{m}$, and blends with PiPrOx and PcycloPrOx were evaluated (Figure 1b,c: for synthesis and material characterization, see Figure S2-S7, Supporting Information; for establishment of MEW, see Figure S8-S11, Supporting Information). Both blends exhibit phase separation (see Supporting Information for details) but show different behavior during processing. Only blends of 10-30\% PnPrOx and 70-90\% PcycloPrOx revealed a thread-like structure composed of crystalline PnPrOx fibrils after the dissolution of PcycloPrOx (Figure 1e,h, Figures S12 and S13, Supporting Information). Melt electrofibrillation of the $30 \% \mathrm{PnPrOx}+70 \%$ PcycloPrOx blend resulted in fibrils with the best stability and handling properties in cell culture (Figure S13, Supporting Information). As documented by SEM, the aligned fibrils were fabricated in reproducible quality with diameters normally distributed around $1.5 \mu \mathrm{m}$ (Figure 1d,g,j). Importantly, the electric field has a dominant effect during processing and is crucial for the formation of uniform and continuous fibrils (Figures S14 and S15, Supporting Information). However, the stability and brittleness of the fibrils based on PnPrOx still impair the usability of these fibrilbased scaffolds. Furthermore, the obtained fibrillar micro-bundles exhibited a similar topography as collagen I (fibril diameter $200 \mathrm{~nm}$, Figure 1d) but cannot really be regarded biomimetic because of the diameter difference.

To ensure better fibril-stability and create fibrils with smaller diameter size for increased collagen I mimicry, we adapted the system to another polymer combination with poly( $\varepsilon$-caprolactone) (PCL) as fibrilling polymer. PCL is especially interesting regarding biofabrication because it is a biodegradable thermoplast that is approved for clinical use with good mechanical stability and can be processed via MEW.[19] We thus screened different blends of PCL with polymers that exhibit selective solubility postprocessing such as Pluronic F-127, PEG 4000, and poly(vinylacetate) (PVAc) (Figure S16, Supporting Information). Here, MEW of $70 \%$ PVAc $+30 \%$ PCL led to the creation of ordered scaffolds. To avoid influence of solvents, we used a compounder for blending the PCL/ PVAc system (Figure S17, Supporting Information). After dissolution of PVAc in $70 \%$ ethanol, highly ordered aligned PCL fibers were exposed (Figure 1e,h, Movie S1, Supporting Information). Blends of PCL and PVAc are known to be miscible under certain conditions ${ }^{[25]}$ in contrast to the immiscible POxbased blends. Unexpectedly, the resulting PCL fibrils showed smaller diameters $(\approx 300 \mathrm{~nm})$ as compared to fibrils based on PnPrOx. This indicates a more complex and subtle dependence of structure formation during melt electrofibrillation. The PCL fibrils exhibit high morphological similarities to natural 
a
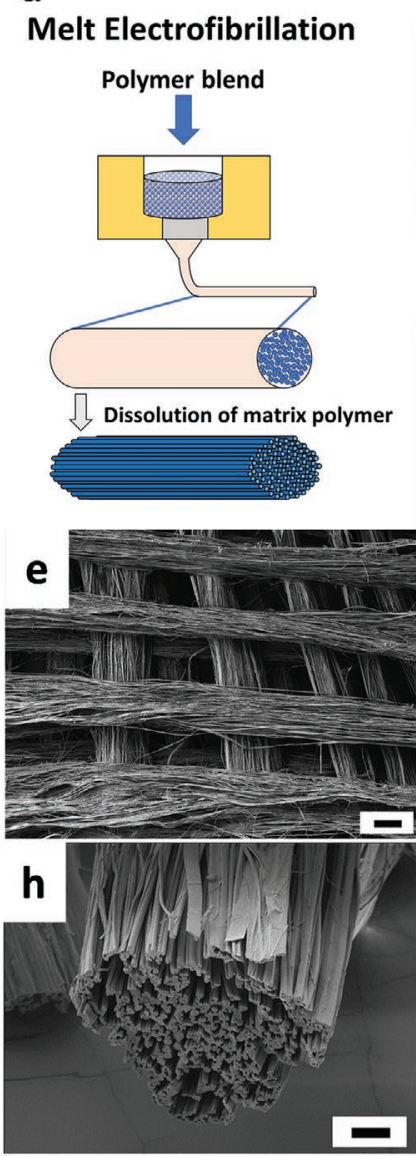

b

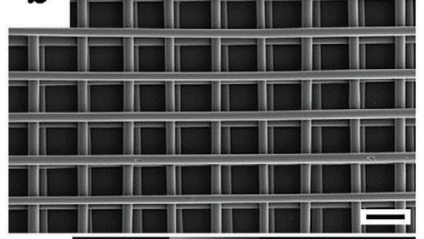

c
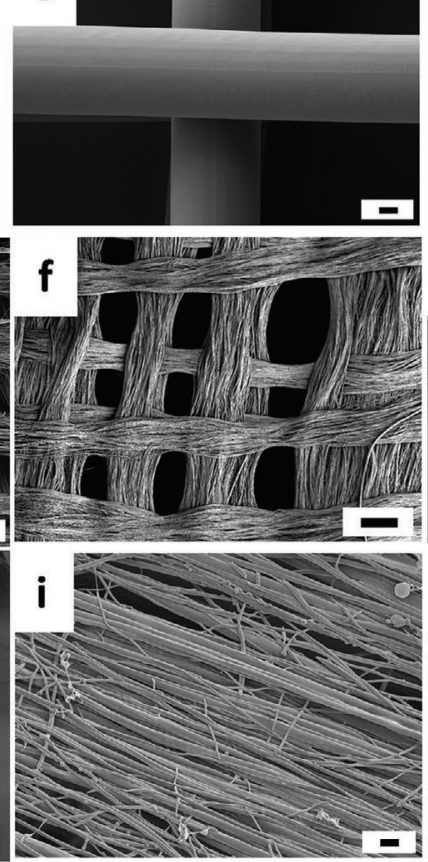

d
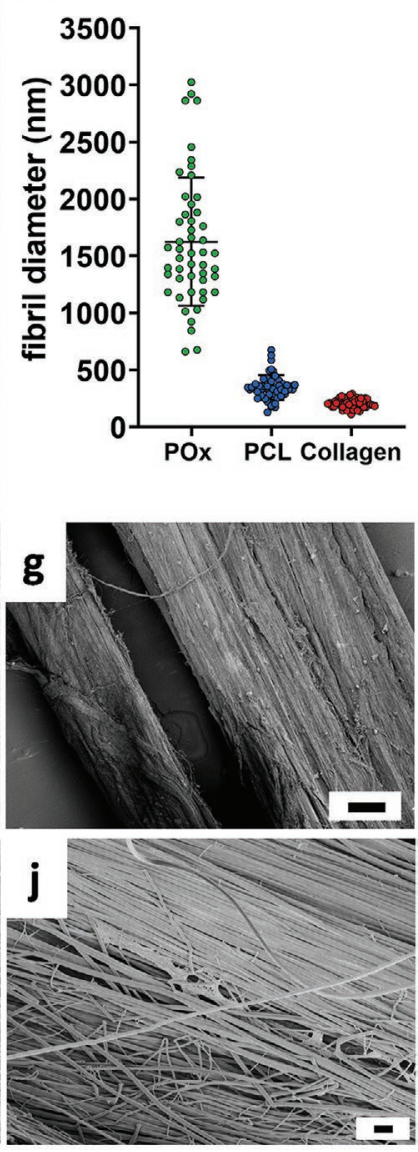

Figure 1. Melt electrofibrillation. a) Manufacturing scheme of fibrillar scaffolds. A polymer blend is processed via MEW. After dissolution of the matrix polymer, aligned nanofiber microbundles emerge. b,c) SEM image of blend scaffold before dissolution of the sacrificial polymer. d) Fiber diameter measurements of POx-, PCL-, and collagen-fibrils (50 values each). e,h) SEM images of PnPrOx fibril scaffold after dissolution of PcycloPrOx. f,i) SEM images of the PCL fibril scaffold after the dissolution of PVAc. g,j) SEM images of rat tail collagen fibrils. c) Fibril diameter size distribution. (scale bars in (b): $200 \mu \mathrm{m}$; in (e-g): $100 \mu \mathrm{m}$; in (c,h): $10 \mu \mathrm{m}$; in $(\mathrm{i}, \mathrm{j}): 1 \mu \mathrm{m})$.

collagen fibrils prepared from decellularized rat tail collagen I (Figure 1f,I,j, Figure S18, Supporting Information) and can thus be regarded truly biomimetic.

\section{Evaluation of Human Macrophage Phenotype on Collagen-Mimetic Nanofiber Microbundles}

Nanofibrillar-microbundle-based scaffolds created by melt electrofibrillation from either 30\% PnPrOx $+70 \%$ PcPrOx blends (POx fibril) or $70 \%$ PVAc $+30 \%$ PCL (PCL fibril) were compared to MEW-fabricated microfiber-based POx or PCL control scaffolds (POx fiber, PCL fiber) that exhibit a smooth surface and a fiber diameter that corresponds to the bundle-diameter of the melt-electrofibrillated scaffolds. POx-coated wells and PCL films were used as further 2D controls for macrophage cultivation (Figure S20a,b, Supporting Information). For direct comparison, the results of the morphological analysis of macrophages on collagen I fibrils were added in Figure 2. The morphology of macrophages, analyzed via actin-staining and SEM after $7 \mathrm{~d}$ of culture, was dependent on the type of scaffold topography.
On smooth fiber scaffolds (Figure 2a,f and c,e), macrophages adopted a larger, roundish, and flattened morphology. In contrast, macrophages on fibril scaffolds (Figure 2b,g, d,e, and e,j) aligned with the direction of the individual fibrils and were able to migrate into and to elongate inside the scaffold (Figure $2 \mathrm{k}$, Movie S2, Supporting Information). While no significant differences were observed for the cellular aspect ratio of macrophage on fiber scaffolds, macrophages on PCL fibrillar scaffolds elongate up to $190 \mu \mathrm{m}$ (average $100 \mu \mathrm{m}$ ), compared to macrophages on POx (average $80 \mu \mathrm{m}$ ) and collagen fibrils (average $50 \mu \mathrm{m}$ ) (Figure 2l-n). On the 2D controls, morphological differences of macrophages were observed. While macrophages on POx coatings adopted a small and spherical phenotype, on PCL films, macrophages appeared in a mixed population of flat, roundish, and slightly elongated cells (Figure S20a,b, Supporting Information).

The gene expression profile of spontaneously differentiated macrophages was analyzed after a culture period of seven days on fiber (POx, PCL) and fibril scaffolds (POx, PCL, collagen) via qPCR (Figure 3a). As further 2D material controls POx coatings, PCL films (Figure S20, Supporting Information) 

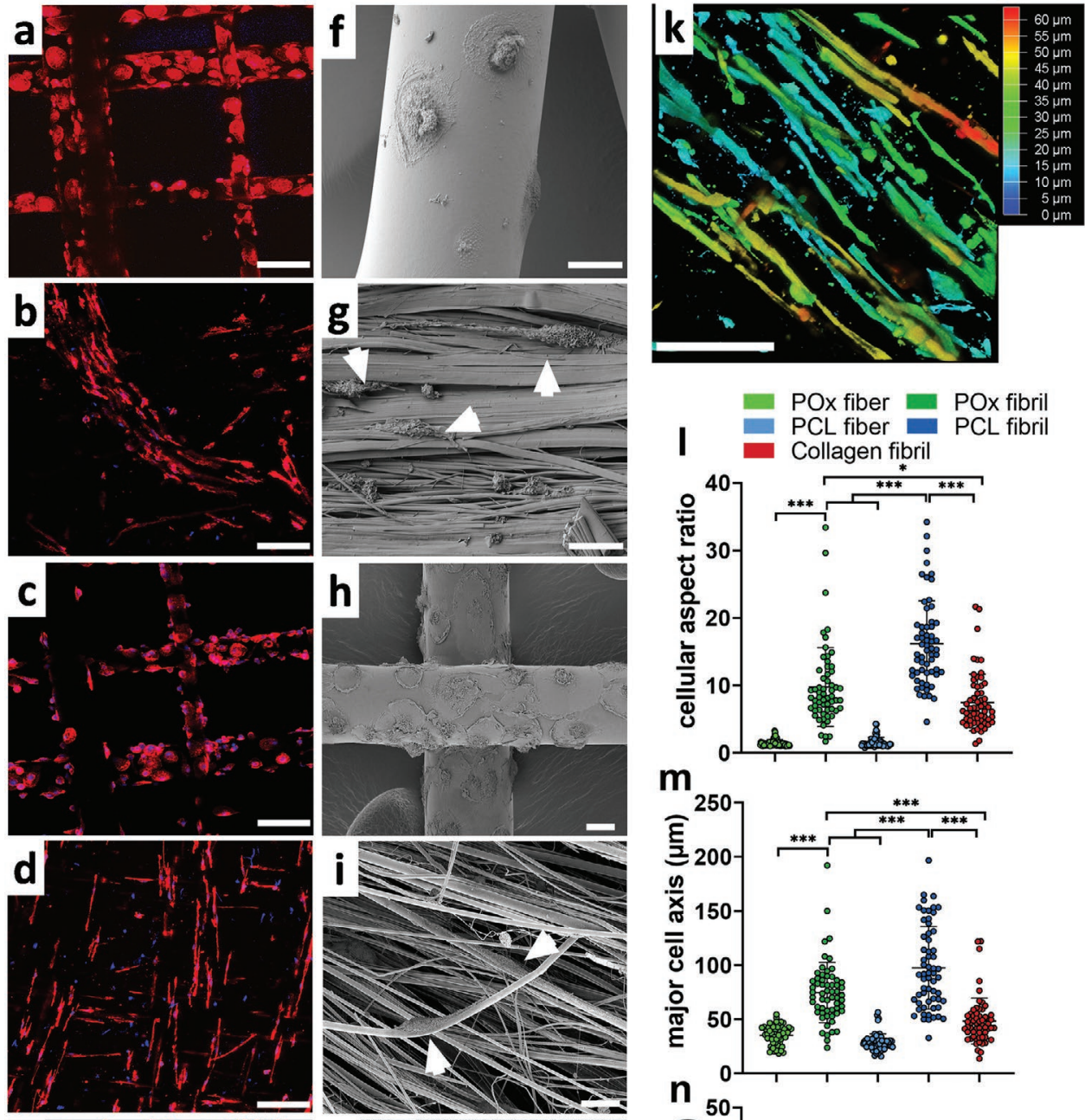

\section{m}

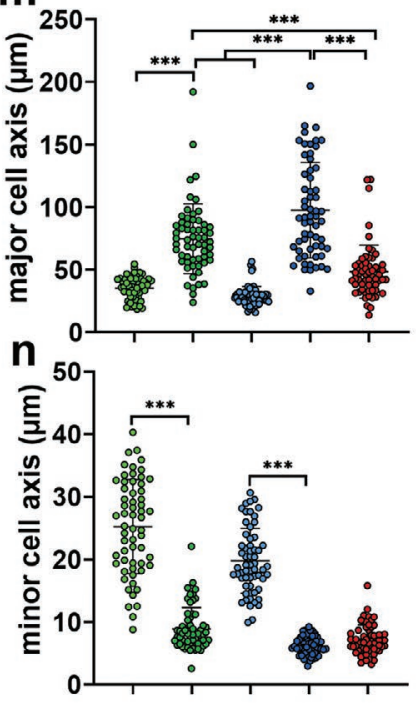

Figure 2. Cellular morphology of human macrophages on fiber and fibrillar scaffolds. a-e) Phalloidin staining of actin filaments (actin: red, nucleus: blue; and f-j) SEM images reveal macrophage morphology after $7 \mathrm{~d}$ on POx smooth fiber (a,f), POx fibrillar (b,g), PCL smooth fiber (c,h), PCL fibrillar $(d, i)$, and collagen (e,j) scaffolds. The white arrowheads indicate elongated cells. k) Depth coding of a phalloidin-stained PCL fibrillar scaffold displays the macrophage distribution within the fibrillar bundle. $\mid-n)$ The cellular aspect ratio of 20 cells per donor $(n=3)$ after $7 \mathrm{~d}$ of culture $(l)$ was calculated by division of the determined major cell axis $(\mathrm{m})$ from the minor cell axis $(\mathrm{n})$. $* p<0.05$; $* * * k<0.001$. (Scale bars in $(\mathrm{a}-\mathrm{e}, \mathrm{k}): 100 \mu \mathrm{m}$; in $(\mathrm{f}-\mathrm{j}): 20 \mu \mathrm{m})$.

and collagen coatings were used. Based on the gene expression pattern of the M2 markers CD206 and IL-10 as well as the M1 markers IL-1 $\beta$ and IL-8, a spontaneous differentiation on all samples compared to the reference samples (corresponding fiber scaffolds (POx, PCL)/coating (collagen)) on day 1 (expression level at 1) were observed.

At day 1, all tested markers were upregulated on fibrillar scaffolds compared to the corresponding control, with the highest expression observed on collagen fibrils. The M2 marker IL-10 was further upregulated on all samples, except for POx fibrils, after $7 \mathrm{~d}$ of culture. In addition, IL-10 was at least twice as strongly expressed on all fibrillar scaffolds on the seventh day as on the corresponding fiber scaffold/coating. Additionally, CD206 was upregulated only on PCL and collagen fibrils. Here, an up to 80-fold upregulation compared to the corresponding control sample was observed. Accordingly, CD206 protein expression was upregulated on PCL fibrillar scaffolds compared to fiber scaffolds (Figure S21d, Supporting Information). In all groups, both M1 markers were downregulated after $7 \mathrm{~d}$, while particularly macrophages cultivated on fiber scaffolds showed 

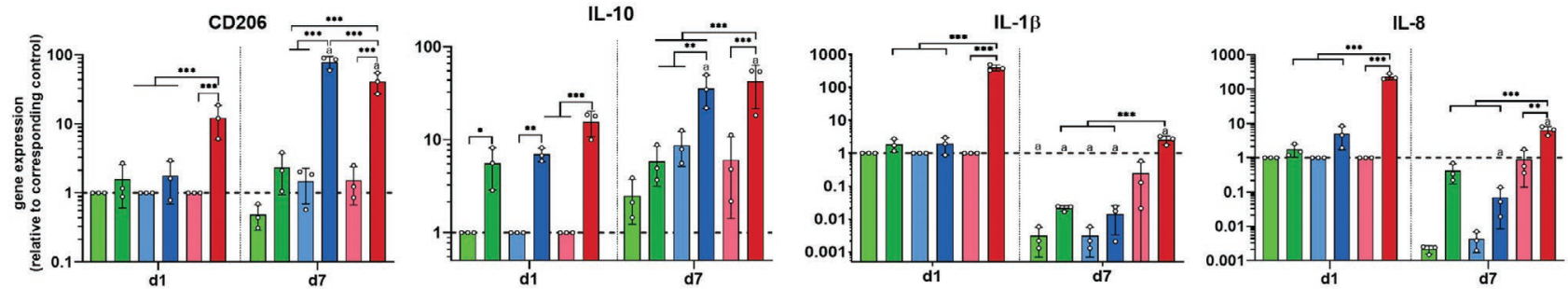

b

IL-10
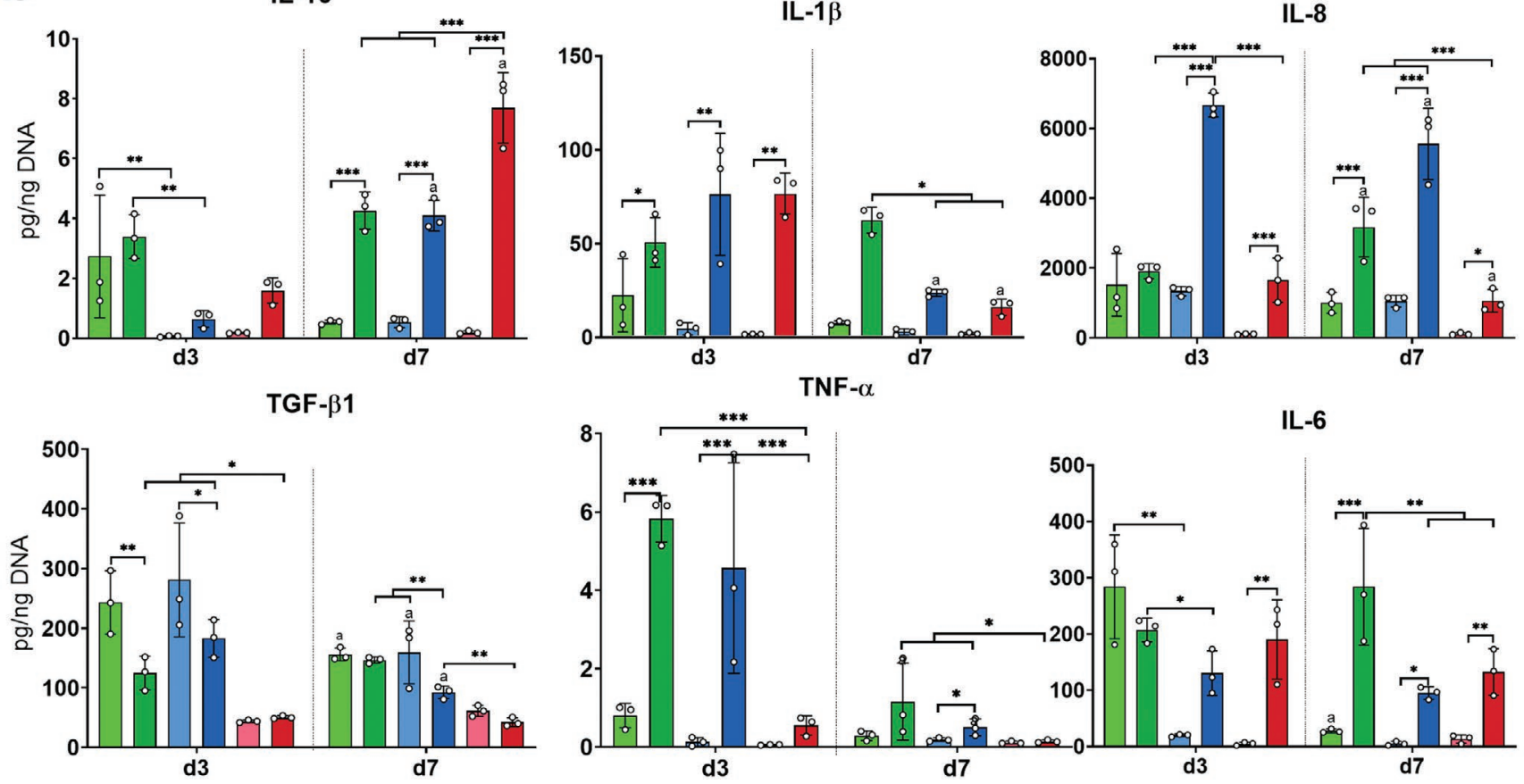

Figure 3. Differentiation patterns of spontaneously differentiated macrophages. a) Gene expression was analyzed via qPCR. Spontaneous differentiation was observed on all tested material groups: 3D fiber (POx, PCL), 3D fibrillar scaffolds (POx, PCL, collagen), and 2D collagen coating, compared to the reference sample (monocytes/macrophages on day 1 on the corresponding material fiber (POx, PCL) or coating (collagen)). To detect changes in macrophage polarization, the gene expression of the M2 markers CD206 and IL-10, as well as of the M1 markers IL-1 $\beta$ and IL-8, was analyzed on days 1 and 7 (mean \pm standard deviation (SD), $n=3$ ). $* p<0.05$; $* * x<0.01$, $* * * p<0.001 ;{ }^{a} p<0.05$ versus d1, same culture condition. b) Cytokine release of IL-10, TGF- $\beta 1$, IL-1 $\beta$, IL-6, IL-8, and TNF- $\alpha$ was measured using supernatants of macrophages cultivated for 3 and $7 \mathrm{~d}$ on 3D fiber (POx, PCL), 3D fibrillar scaffolds (POx, PCL, collagen) and 2D collagen coating. Amount of released cytokine was normalized to the DNA, determined of the same scaffold. (mean $\pm \mathrm{SD}, n=3$ ). $* p<0.05 ; * * p<0.01$, * * ${ }^{*} p<0.001 ;{ }^{a} p<0.05$ versus $\mathrm{d} 3$, same culture condition.

lower expression compared to those cultivated on fibrillar scaffolds.

Spontaneously differentiated monocyte-derived macrophages were tested for their cytokine release of IL-10, TGF- $\beta 1$, IL- $1 \beta$, TNF- $\alpha$, IL-6, and IL- 8 , into the cell culture medium after 3 and $7 \mathrm{~d}$ (Figure 3b). On day 7, the release of the M2 marker IL-10 was significantly upregulated on the fibrillar scaffolds compared to fiber scaffolds. Furthermore, on day 3, IL-10 was significantly more highly released by macrophages cultivated on POx samples. While in general, the fibrotic-related M2 marker TGF- $\beta 1$ was released lowest on collagen samples compared to the synthetic materials, no significant differences between the 3D and 2D collagen samples were observed. Contrarily, on POx and PCL fibrils, TGF- $\beta 1$ was significantly lower than on fiber scaffolds.

Interestingly, the release of the M1 markers IL-6, IL-8, IL- $1 \beta$, and TNF- $\alpha$ was lowest on fiber scaffolds with a decrease or remained low level over the whole culture period. In contrast, on fibril scaffolds, the M1 markers IL- $1 \beta$ and TNF- $\alpha$ were released on high levels on day 3 and significantly decreased over time. In contrast, the release level of the markers IL- 8 and IL-6 was maintained.

The above-described different morphology between macrophages on 2D POx and PCL controls could also be shown for the polarization outcome (Figure S20c,d, Supporting Information). Macrophages cultivated on PCL films showed a 
a
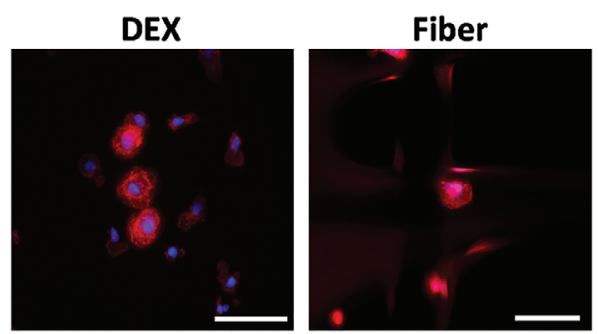

b

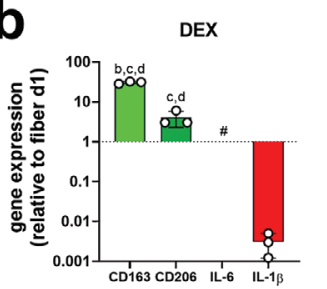

C
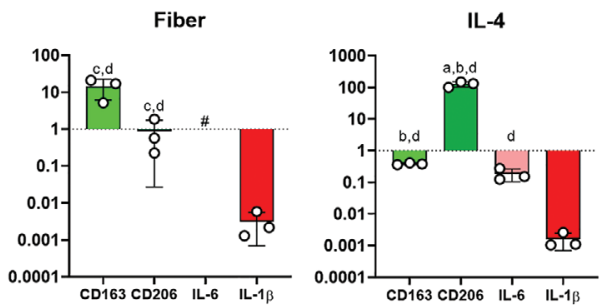

Fibril
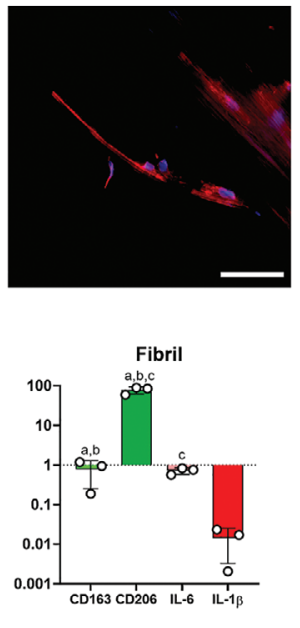

Collagen I fibril

Cell morphology

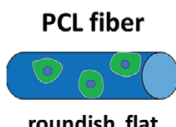

PCL fibril

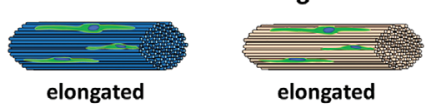

elongated

\begin{tabular}{|c|c|c|c|c|c|c|c|}
\hline \multicolumn{2}{|c|}{ Gene Expression (e) /Release ( $r$ ) } & d1/d3 & \multirow{2}{*}{\begin{tabular}{|c|} 
d7 \\
$14( \pm 6.7)$
\end{tabular}} & \multirow{2}{*}{$\frac{d 1 / d 3}{1,6( \pm 0.7)}$} & \multirow{2}{*}{$\frac{d 7}{0,7( \pm 0.4)}$} & \multirow{2}{*}{$\frac{\mathrm{d} 1 / \mathrm{d} 3}{4.6( \pm 2.1)}$} & \multirow{2}{*}{$\frac{d 7}{5.8( \pm 3.2)}$} \\
\hline $\begin{array}{l}\text { Anti-inflammatory/ } \\
\text { Pro-healing }\end{array}$ & $\begin{array}{l}\text { CD163 } \\
\text { (e) }\end{array}$ & 1 & & & & & \\
\hline & \begin{tabular}{|l|} 
CD206 \\
(e) \\
\end{tabular} & 1 & $1.5( \pm 0.6)$ & $1.7( \pm 0.9)$ & $78( \pm 13.2)$ & $12.2( \pm 5.1)$ & $41( \pm 11.2)$ \\
\hline & $\begin{array}{l}\text { IL-10 } \\
\text { (r) }\end{array}$ & $0.06( \pm 0.02)$ & $0.5( \pm 0.15)$ & $0.6( \pm 0.22)$ & $4.1( \pm 0.4)$ & $1.5( \pm 0.34)$ & $7.6( \pm 0.96)$ \\
\hline Pro-fibrotic & \begin{tabular}{|l} 
TGF- $\beta$ \\
$(r)$ \\
\end{tabular} & $280( \pm 77)$ & $160( \pm 43)$ & $183( \pm 25)$ & $92( \pm 8.6)$ & $76( \pm 8.9)$ & $42( \pm 6)$ \\
\hline \multirow[t]{2}{*}{ Pro-inflammatory } & \begin{tabular}{|l|} 
IL-1B \\
$(r)$ \\
\end{tabular} & $4.6( \pm 2.6)$ & $2.9( \pm 1.3)$ & $76( \pm 26)$ & $24( \pm 1.5)$ & $76( \pm 8.9)$ & $16( \pm 3.4)$ \\
\hline & \begin{tabular}{|l|} 
TNF- $\alpha$ \\
$(r)$ \\
\end{tabular} & $\begin{array}{r}0.13( \pm \\
0.11)\end{array}$ & $0.19 \pm 0.05)$ & $4.6( \pm 2.1)$ & $0.5( \pm 0.13)$ & $0.5( \pm 0.9)$ & $0.14( \pm 0.03)$ \\
\hline Pro-angiogenic & \begin{tabular}{|l} 
IL-8 \\
$(r)$
\end{tabular} & $\begin{array}{r}1336.5( \pm \\
109.1) \\
\end{array}$ & $1050( \pm 189)$ & $6672( \pm 277)$ & $5561( \pm 883)$ & $1653( \pm 516)$ & $1059( \pm 263)$ \\
\hline $\begin{array}{l}\text { Similarities to } \\
\text { macrophage-type } \\
\text { (function, stimuli) }\end{array}$ & & \multicolumn{2}{|c|}{$\begin{array}{c}\text { M2c } \\
\text { (deactiviated, } \\
\text { DEX-induced }\end{array}$} & \multicolumn{2}{|c|}{$\begin{array}{c}\mathrm{M} 2 \mathrm{a} \\
\text { (tissue regeneration, } \\
\text { IL-4-induced) }\end{array}$} & \multicolumn{2}{|c|}{$\begin{array}{c}\text { M2a } \\
\text { (tissue regeneration, } \\
\text { IL-4-induced) }\end{array}$} \\
\hline
\end{tabular}

Figure 4. Comparison of chemically and physically induced macrophage phenotypes. Macrophages were either cultivated in the presence of dexamethasone (DEX) or IL-4 on polystyrene, or on PCL fiber or fibrillar scaffolds. a) Phalloidin staining of the actin fibers after $7 \mathrm{~d}$ of cultivation (actin: red, nucleus: blue). b) Gene expression analyzed via qPCR. The M2 marker CD163 and CD206, as well as IL-6 and the M1 marker IL-1 $\beta$, were analyzed on day 7. Data are normalized to macrophages cultivated on PCL fiber scaffolds on d1 (mean \pm SD, $n=3$ ). ${ }^{a} p<0.05$ : relative to DEX; ${ }^{b} p<0.05$ : relative to fiber; ${ }^{c} p<0.05$ : relative to IL-4; ${ }^{d} p<0.05$ : relative to fibril. c) Overview of the macrophage response to PCL microfibers and nanofibril-bundles and collagen I nanofibril bundles. Alterations in macrophage morphology, gene/protein expression, and cytokine release dependent on topographical cues over seven days compared to the first (gene expression (e))/third day (cytokine release (r)) are displayed. Significant changes $(p<0.05)$ are indicated in green (upregulation) and red (downregulation).

polarization ranging between that of macrophages cultivated on PCL fiber and fibril scaffolds. Instead, 2D POx coatings induced higher M1 marker expression (IL-1 $\beta$ and IL-8) and lower expression of M2 markers (CD163, CD206, IL-10) as fiber and fibrillar POx scaffolds.

Based on the previous results, two distinct macrophage phenotypes were observed depending on the scaffold topographies differing in morphology and polarization. Since similar characteristics are known for in vitro chemically induced M2 macrophage phenotypes, ${ }^{[15]}$ we were interested in investigating whether there is a correspondence between physically (PCL scaffold topographies) and chemically (M2) stimuli (IL-4 (M2a) and dexamethason (DEX) (M2c); macrophages cultured on polysterene) activated human macrophages.

By actin-staining, morphological differences of monocytederived macrophages after treatment with Dex and IL-4 were observed (Figure 4a). While M2c macrophages had a predominantly roundish morphology with a diameter of around $25 \mu \mathrm{m}$, IL-4 induces a mixture of smaller, roundish cells, but also of elongated macrophages. As already shown in Figure 2, the cultivation of macrophages on fiber scaffolds adopted a phenotype similar to that induced by DEX. In contrast, macrophages 
cultivated on fibril scaffolds were more similar to the chemically induced M2a macrophages.

Gene expression analysis revealed further similarities between chemical and physical induction (Figure 4b). While DEX and fiber scaffolds induced the upregulation of the M2 marker CD163, macrophages treated with IL-4 and macrophages cultivated on fibril scaffolds more highly expressed the M2 marker CD206. IL-4 induced M2a macrophages are known to upregulate IL-6, which in this case acts as an antiinflammatory marker. ${ }^{[26]}$ Although our data showed that IL-6 was downregulated in IL-4 and fibril-induced macrophages compared to the reference sample (fiber d1), it was still measurable. Instead, M2c macrophages and those cultivated on fiber scaffolds did not express IL-6 on day 7 at all. Additionally, all four groups downregulated the M1 marker IL-1 $\beta$.

For better comparison, the results of the macrophage analysis were summarized in Figure $4 \mathrm{c}$ and with added absolute/ relative data in Figure S24 (Supporting Information). The results show high similarity between macrophages cultivated on PCL and collagen I fibrillar scaffolds.

\section{Discussion}

Since aligned structured collagen I fibrils are the major part of the $\mathrm{ECM}^{[18]}$ and collagen I is involved in every phase of wound healing where macrophages are key players, ${ }^{[27]}$ we examined the behavior of human macrophages on aligned structured collagen I fibrils. Our results showed elongation of macrophages correlated with an M2-like polarization with similar efficiency and intensity comparable to the standard biochemical stimulation by IL- 4 and thus indicated the importance and potential role of this effect in tissue regeneration healing. As this effect did not occur on 2D collagen I coatings, we demonstrated the induction by the hierarchical fibrillar structure.

To prove the material independence, we developed melt electrofibrillation as additive manufacturing like fabrication method for customized scaffolds composed of collagen I inspired but synthetic polymer-based fibril bundles. This method is technologically based on melt electrowriting as solvent-free processing technology of medical-grade thermoplastics such as poly(e-caprolactone) (PCL). ${ }^{[28]}$ Using an appropriate mixture of nonmiscible blends instead of homopolymers, flow-induced oriented phase separation is induced during processing that yields an internal structure of the fabricated filament. Suited combinations and volume ratios of the two polymers lead to the formation of ultrathin fibrillary structures of the lower volume share polymer in the matrix of the higher volume share thermoplastic. The crucial step to reveal nanofibrillar bundles of the lower volume share polymer (fibrilling polymer) remain is to select the thermoplastics appropriately so that the volume polymer can selectively be dissolved after solidification. We successfully established this procedure for two different polymer blends: PnPrOx + PcycloPrOx and PCL + PVAc, both in the ratio of $30 \mathrm{vol} \%$ to 70 vol\% (for details see Supporting Information).

The medical-grade-based PCL fibrillar scaffolds consist of a clinically approved polymer and are structurally more stable with good handling properties compared to the brittle POxbased scaffolds. Importantly, the PCL fibrils can be fabricated with a decreased average fibril diameter down to $300 \mathrm{~nm}$ as compared to $1500 \mathrm{~nm}$ for PnPrOx fibrils. Our measurements showed that dry collagen fibrils have a diameter of about $150-200 \mathrm{~nm}$, which is in accordance with other studies. ${ }^{[29]}$

Therefore, our fabricated PCL fibrils are thus not only more stable and better to handle but can be described as structurally truly biomimetic.

PCL and PnPrOx polymeric scaffold topographies revealed changes in cell morphology and expression pattern of human macrophages. On microfilament, fiber scaffolds produced by MEW that exhibit a similar filament diameter as the bundle of nanofibrils produced by melt electrofibrillation, macrophages adopted a roundish morphology and showed similarities to Dex-induced M2c ("deactivated") macrophages, ${ }^{[15]}$ characterized by the inhibition and downregulation of M1-related markers. On fibrillar-based scaffolds, macrophages exhibited a pronounced elongation, particularly on PCL.

Geometrical confinement in two and three dimensions is an important driver for macrophage polarization. It has been shown that preventing rodent macrophage spreading via micropatterning or microwells leads to downregulation of inflammation-associated transcriptional programs, ${ }^{[30]}$ which has also been shown by adapting pore sizes in electrospun scaffolds. ${ }^{[31]}$ Along that line, it was further reported that macrophage polarization is affected by topographical patterns differently than for flat substrates, both for rodent macrophages ${ }^{[12]}$ and also for human-monocyte-derived macrophages. ${ }^{[32]}$ Vassey et al. reported that denser patterns promote the M2 polarization of human-monocyte-derived macrophages, ${ }^{[33]}$ which demonstrates that the dimensions of geometrical patterns are important in this context.

Also the immune response to biomaterials in rodent in vivo models is significantly affected through topographical patterns ${ }^{[33,34]}$ and also in sphere-templated hydrogels with highly regular and interconnected pores. ${ }^{[13]}$ Moreover, for fibrous scaffolds, fiber diameters of around $500 \mathrm{~nm}$ minimized the immune response of murine macrophages compared to microfibrous scaffolds $(\approx 1.5 \mu \mathrm{m}) \cdot{ }^{[35]}$ Coherently, it has been shown that the possibility for cells to penetrate scaffolds plays an important role for improved tissue repair. ${ }^{[36]}$ In this context, Jiang et al. created random and aligned expanded electrospun scaffolds, which allowed cell penetration based on the introduction of pores and compared them to non-expanded electrospun scaffolds in an in vivo study. ${ }^{[37]}$ Increased macrophage infiltration was found in the expanded scaffolds, accompanied by a regenerative response and increased neovascularization. Importantly, the expanded, aligned scaffolds showed thinner fibrous capsules and the lowest amount of infiltrated giant cells compared to the random expanded scaffolds, highlighting the importance of alignment and penetrability as scaffold design criteria for biomaterial-based immunomodulation.

While most of the literature deals with rodent macrophage cell lines (e.g., RAW) or primary rodent immune cells, significantly less studies deal with primary human immune cells. This is remarkable, since on the one side, effects that are observed for human immune cells in vitro do translate to rodent in vivo models, ${ }^{[33,34]}$ and on the other side, human immune cells are, of course, directly relevant for the eventual organism of interest. It appears that many stimuli beyond soluble factor stimulation 
lead to significant effects in rodent immune cells but to less pronounced or even no effects in human immune cells. One of these stimuli is the topography-induced cellular elongation, which has been described for rodent macrophages based on micro and nanopatterning of 2D substrates or thin layers of electrospun meshes already in 2010 and 2013, respectively. ${ }^{[12,32]}$ The first study that showed a little pronounced elongation of human immune cells was our previous study in which we used melt electrowritten scaffolds prepared from PCL with fibers in rectangular patterns, where interfilament spacings of $40 \mu \mathrm{m}$ resulted in a certain elongation of human macrophages and a resulting mild phenotype drift towards M2. ${ }^{[14]}$ However, the cells did not experience significant spatial confinement, and the resulting effect was not very pronounced.

In the present study, human primary monocyte derived macrophages were able to migrate into the fibrillar bundles (Figure $2 \mathrm{k}$ ). In fact, only a few cells remained directly visible at the surface of the bundles, and the fibrillar bundles had to be opened for cell visualization. Obviously, this particular topography stimulated the cells to invade and to remain inside.

Inside the fibrillar bundles, the cells are within a truly $3 \mathrm{D}$ and highly anisotropic, oriented spatial confinement. Macrophages showed less secretion of IL- $1 \beta$ and TNF- $\alpha$ on the PCL fibrillar scaffolds than on the POx fibrillar scaffolds that exhibited a larger fibril diameter, which correlates well with the abovementioned literature data for murine cells. ${ }^{[35]}$ Since the intensity of the elongation and subsequent polarization was highest on collagen I fibrils and lower on the PnPrOx scaffolds than on PCL, with the PCL fibrils resembling the collagen I fibrils more closely, the effect intensity correlates with the structures' biomimicry, which supports the hypothesis of the biological relevance of this effect.

In contrast to murine macrophages ${ }^{[12]}$ so far, the elongation of human macrophages in 2D was rather related to an induced M1 differentiation. ${ }^{40]}$ However, we showed that also human IL-4 induced M2a macrophages tend to be more elongated than noninduced macrophages in 2D (Figure S22, Supporting Information). Moreover, the expression pattern, especially the upregulation of CD206 ${ }^{[15]}$ and IL-6 ${ }^{[26]}$ and repressing of CD163, ${ }^{[41]}$ indicates a similarity of elongated human macrophages on fibrillar scaffolds to M2a, IL-4 induced macrophages, which are considered to be involved in tissue regeneration and repair processes. ${ }^{[15]}$ Although the polarization by fibrillar topography and IL-4 induction might be regulated differently by cytoskeleton dependent or independent mechanisms (Figure S23, Supporting Information), we take this as another argument that the biomimetic 3D fibrillar structure is key to a pro-healing response for human macrophages.

Additionally, fibrillar scaffolds, particularly PCL and collagen I, induced a significant M1-M2 switch with strong upregulation of the M1 markers IL- $1 \beta$ and TNF- $\alpha$ on early time points, compared to smooth fiber scaffolds. After $7 \mathrm{~d}$, those M1 markers were downregulated, accompanied by upregulation and secretion of the M2 markers CD206 and IL-10, which we refer to as M2 transition in this study. Proper wound healing after injuries or implantations of biomaterials requires the involvement of both M1 and M2 macrophages, however, in an ordered timeline. While the absence of M1 macrophages in the early stages of regeneration impairs healing ${ }^{[42]}$ and could affect proper angiogenesis, ${ }^{[43]}$ a prolonged occurrence can lead to chronic inflammation or fibrosis. ${ }^{[4]}$ Therefore, a biomaterial should not inhibit the early M1 polarization but induce a punctual phenotypical switch. The fibrillar scaffolds in our study induced a significant M1-to-M2 switch in human macrophages that resembles the transient phenotype shift that is characteristic for normal wound healing. We suggest that the migration into the fibrillar bundles and the elongation helps to generate this M1M2 transition and that similar processes take place in vivo. In this context, it is important to note that the upregulation of IL-8 on fibrillar scaffolds might not fit the standard definition of an M2 transition. However, IL-8 fulfills important functions in angiogenesis, ${ }^{[45]}$ which is an important feature of healing and might be beneficial for biomaterial integration. ${ }^{[46]}$

It is known that the chemical composition of polymeric materials, ${ }^{[34]}$ and the protein corona that is resulting after contact with biological fluids, ${ }^{[38]}$ do influence the polarization state of macrophages. In our study we used human platelet lysate as a culture medium, which we have established before as suited in vitro condition for human macrophage culture ${ }^{[39]}$ as a condition that is as close as possible to the early time points of materialhost contact in vivo. We compared the highly oriented fibrils for the two synthetic polymers and collagen I with flat films, and for the two polymers also with filaments with a diameter of the fibrillar-bundles, always under platelet lysate conditions. The data show pronounced effects only for the fibrillar samples, which clearly underlines that the collagen-mimetic topography triggers them.

Taken together, we are confident that the observed strong effect on human macrophage polarization is triggered and driven by the highly anisotropic spatial confinement of the cells, and that the collagen I fiber mimicry is key to this effect. We further hypothesize that the monocyte-derived macrophage interaction with collagen I fibrils is thus of biological relevance as a part of the natural healing cascade.

We further demonstrated that human macrophage elongation led to lower TGF- $\beta 1$ release than a roundish morphology on fiber scaffolds. Recent studies showed that a denser collagen network was related to a decreased expression of TGF- $\beta 1$ by macrophages with following decreased induced differentiation of fibroblasts into myofibroblasts, which are major contributors of collagen. ${ }^{[47]}$ Thus, already present collagen-like fibril structures, as of our scaffolds, might influence the secretion of TGF- $\beta 1$ of macrophages to reduce further collagen deposition, leading to a decreased fibrotic reaction.

\section{Conclusion}

This study evidences the biological relevance of collagen I fibrils as structural motifs for human macrophage elongation induced phenotype shift. We demonstrate that this effect is driven by the ECM mimicking fibrillar 3D topography and comparable in strength and expression pattern to standard cytokineinduced immunomodulation. To prove the mere topographic nature of this effect, we developed melt electrofibrillation as a novel fabrication technology, which allows fabricating structurally collagen-mimetic nanofibrillar microbundles with the entire freedom of scaffold design that 3D printing offers. These 
aligned fibril bundles allow cell infiltration and provide an environment that prevents unnatural apical/basal polarization by providing truly 3D topographical cues for the infiltrated cells leading to efficient M2-like phenotype polarization like collagen I fibrils that showed similarities to the established IL-4-based biochemical induction. Thus, this study delivers first clear evidence that structural elements of the ECM possess potent topographic immunomodulatory capacity that can be translated into biomaterials for the endowment of truly biomimetic scaffolds and implants with prohealing capacity.

\section{Experimental Section}

Production of MEW Fibers/Scaffolds Fabricated with PnPrOx, PcycloPrOx, and their Blend: To create blends with different ratios of $\mathrm{PnPrOx}$ and PcycloPrOx, $1 \mathrm{~g}$ of each of the polymers was dissolved in $50 \mathrm{~mL}$ deionized water and lyophilized (Synthesis and characterization Figures S2-S7 and Tables S1 and S2, Supporting Information).

For the process of melt electrowriting, a custom-built device was used already described elsewhere. ${ }^{[48]}$ In order to produce homogenous fibers, the MEW device was equipped with one heating zone at the syringe and another one at the nozzle. The process parameters for MEW of PnPrOx are described in Table S3 (Supporting Information).

The same process parameters were used for PcycloPrOx as well as for the polymer blend, except for the temperature of the syringe, which was increased to $210{ }^{\circ} \mathrm{C}$ while the temperature of the nozzle was $225^{\circ} \mathrm{C}$. Besides the standard collector plate, a heated collector plate connected to the temperature control device C 448 by Hotset (Lüdenscheid, Germany) was used. The $\mathrm{G}$ code used for fiber deposition via MEW is available in the SI (see G-Code section in the Supporting Information). $\mathrm{PnPrOx}$ and the PnPrOx + PcycloPrOx blend scaffolds were finally crystallized by prolonged heating at $60{ }^{\circ} \mathrm{C}$ for $24 \mathrm{~h}$. Before applied in cell culture, scaffolds were sterilized by UV-treatment $(254 \mathrm{~nm})$ with a UV-lamp (VL-4.LC, Vilber Lourmat, Essen, Germany).

2D controls were created by dissolving 0.1 wt\% PnPrOx in water. $200 \mu \mathrm{L}$ was added in each well of a 24 well plate, dried, and heated at $60{ }^{\circ} \mathrm{C}$ for $24 \mathrm{~h}$ to create crystalline PnPrOx-coatings.

Production of PCL-Fibrils Based on PCL/PVAc Blends: 70\% PVAc (Kremer Pigmente $\mathrm{GmbH} \&$ Co. KG, Aichstetten, Germany) and 30\% PCL (Corbion Purac, USA) were dissolved in dichloromethane (DCM) for $1 \mathrm{~h}$ under stirring conditions. Films of the blend were cast, and DCM evaporated overnight. Afterward, residual DCM was removed via ultrahigh vacuum. Furthermore, the blends were also created in the compounder Xplore (Xplore Instruments BV) at $120^{\circ} \mathrm{C}, 100 \mathrm{rpm}$ for $60 \mathrm{~min}$.

For MEW, the polymer was heated in the syringe to $150{ }^{\circ} \mathrm{C}$, with the nozzle being heated to $165^{\circ} \mathrm{C}$. The scaffolds were created with the same $G$ code used for the POx blends. To prevent delamination of the fibrils, the edges of the scaffolds were pressed together with a heated aluminum stamp. Before application in cell culture, the PCL fibrils were exposed by washing the scaffolds $3 \times$ for $30 \mathrm{~min}$ in $70 \%$ ethanol. This led to the selective dissolution of PVAc, while sterilizing the scaffolds at the same time. Afterward, the scaffolds were washed $3 x$ in sterile PBS for 5 min each.

Determination of the Fiber Diameters: Fiber diameters were determined using the straight-line selection tool of FIJI software. For this, 50 randomly selected fibers were measured from SEM images, and mean values were calculated.

Cell Culture: All experiments were approved by the Local Ethics Committee of the University of Wuerzburg.

Monocytes were isolated from human-blood-derived buffy coats (Blood Donor Service, German Red Cross, Wiesentheid, Germany) of healthy donors with informed consent. Peripheral blood mononuclear cells were collected by density gradient centrifugation with Pancoll (density: 1,077 $\mathrm{g} \mathrm{L}^{-1}$; Pan-Biotech, Aidenbach, Germany). Monocytes were then isolated via negative selection (Pan Monocyte Isolation Kit, Miltenyi Biotec, Gladbach, Germany) and cultivated for up to $7 \mathrm{~d}$ in macrophage culture medium (RPMI-1640, GlutaMAX medium (Thermo Fisher Scientific, Waltham, USA) with $10 \%$ of human platelet lysate ${ }^{[39]}$ ( $\mathrm{hPL}, \mathrm{PL}$ Bioscience, Aachen, Germany) and $1 \%$ penicillin-streptomycin (5000 $\mathrm{U} \mathrm{mL}^{-1}$ ) (Thermo Fisher Scientific, Waltham, USA) in a humidified atmosphere at $37{ }^{\circ} \mathrm{C}$ and $5 \% \mathrm{CO}_{2}$ without any medium change and without additional differentiation factors (unless stated otherwise).

Freshly isolated monocytes were seeded in a $50 \mu \mathrm{L}$ suspension with $0.75 \times 10^{6}$ cells on top of each sample in a tissue culture-treated 24 well plate. For cultivation on coatings, $1 \mathrm{~mL}$ macrophage culture medium was added immediately after cell seeding. To ensure better adherence, cells on scaffolds were preincubated for $0.5 \mathrm{~h}$, before adding an additional cell culture medium.

For experiments requiring induced differentiation, macrophages were cultured either in the presence of $20 \mathrm{ng} \mathrm{mL}^{-1} \mathrm{IL}-4$ for one day prior to analysis or $10^{-7} \mathrm{M}$ dexamethasone over the entire culture period.

Gene Expression Analysis: To ensure that only macrophages cultured on the scaffold were considered, the macrophage-loaded scaffolds were transferred into $1.5 \mathrm{~mL}$ reaction tubes before RNA isolation. Total cellular RNA of macrophages was isolated using PeqGold Trifast (VWR, Darmstadt, Germany) according to the manufacturer's protocol after 1 and $7 \mathrm{~d}$ of monocyte-derived macrophage cultivation. Afterward, cDNA was generated with the high-capacity cDNA Reverse Transcription Kit (Thermo Fisher Scientific) according to the manufacturer's manual. The gene expression levels of macrophages were analyzed via quantitative real-time PCR ( $\mathrm{PPCR}$ ) (StepOnePlus; Thermo Fisher Scientific) with Sybr Select Mastermix (Thermo Fisher Scientific) as described previously. ${ }^{[39]}$ The specific primers used are listed in Table S4 (Supporting Information). RPS27A served as house-keeping gene for normalization. As a reference sample, $1 \mathrm{~d}$ cultured macrophages on the respective material were used.

Analysis of Cytokine Release: To ensure that only cytokines from adherent macrophages were measured, the cell media were collected $24 \mathrm{~h}$ prior to analysis, sterile filtered, and returned to the cultured cells. Additionally, if the cells were cultivated on scaffolds, the scaffolds were transferred to a new well $24 \mathrm{~h}$ prior to the timepoint of analysis.

Cytokine release of monocytes/macrophages was tested via Single Analyte Elisarray Kits (Qiagen, Hilden, Germany) after one, three, and $7 \mathrm{~d}$ of cultivation. The production of IL-1 $\beta$, IL-6, IL-8, IL-10, TNF- $\alpha$, and TGF- $\beta$ was analyzed in supernatants according to the manufacturer's protocol. The absorbance was measured on a plate reader (Tecan, Männedorf, Switzerland) at $450 \mathrm{~nm}$ and corrected by the absorbance at the reference wavelength of $570 \mathrm{~nm}$. The release was normalized to the corresponding determined DNA amount (Figure S18, Supporting Information).

SEM Sample Preparation: For SEM preparation, after $7 \mathrm{~d}$ of cultivation, samples were fixed with $6 \%$ glutaraldehyde for $15 \mathrm{~min}$ on ice. Since PnPrOx dissolves in both acetone and ethanol, the samples were washed with deionized water and frozen in liquid nitrogen. After lyophilization, the samples were fixed on stubs and coated with a $2 \mathrm{~nm}$ platinum layer by a Leica EM ACE600 sputter coater (Leica Microsystems, Wetzlar, Germany).

$\mathrm{PCL}$ and collagen samples were incubated two times with PBS on ice before their dehydration by a graded ethanol series $(2 \times 70 \%, 2 \times 90 \%$, $2 \times 100 \%(\mathrm{v} / \mathrm{v}))$. After drying via hexamethyldisilazane, the samples were fixed and coated as described above.

Phalloidin Staining of Actin Fibers: For staining, macrophages were cultured either on the scaffolds or well plates for $7 \mathrm{~d}$ and fixed with $4 \%$ formaldehyde for $2 \mathrm{~h}$ at room temperature (RT). The samples were washed with $\mathrm{PBS}^{-}$, followed by blocking with $2 \%$ bovine serum albumin in PBS- for 30 min at RT. Phalloidin-iFluor 555 Reagent (Abcam, Cambridge, UK) was applied for $1 \mathrm{~h}$ at RT in a humidified chamber. Subsequently, samples were washed and mounted with "Immunoselect Antifading Mounting Medium" with DAPI (Dianova, Hamburg, Germany). Images were captured via fluorescence microscopy (Axio Observer, Zeiss equipped with epifluorescence optics and a MRm camera; Zeiss, Oberkochen, Germany) or confocal microscopy ((TCS SP8, Leica, Wetzlar, Germany). 
The cellular aspect ratio was calculated by the division of the majo from the minor cell axes, which were measured using the straight-line selection tool of FIJI software. Each 30 cells per donor were measured $(n=3)$.

Statistics: The determination of the statistical significance was performed by the two-way analysis of variance (Anova) using the Statstica 13 software (Statsoft, Hamburg, Germany). Results were considered to be significantly different at a $p$-value below 0.05 . Data were tested for normality and homogeneity of variance.

\section{Supporting Information}

Supporting Information is available from the Wiley Online Library or from the author.

\section{Acknowledgements}

M.R. and T.T. contributed equally to this work. This work was supported by the European Research Council (ERC) within the project Design2Heal (grant number 617989) and the German Research Foundation (DFG) within the collaborative research center TRR 225 (project number 326998133 - subproject B02). Moreover, the authors greatly appreciate the financial support of the Julius-Maximilians University Würzburg, Germany and of the State of Bavarian (Bavarian Gender Equality Grant (BGF)). Special thanks go to Julia Hümmer for initial support with MEW of POx and Paul D. Dalton as well as Tomasz Jüngst and Tatjana Schilling for helpful discussions.

Open access funding enabled and organized by Projekt DEAL.

\section{Conflict of Interest}

The authors declare no conflict of interest.

\section{Data Availability Statement}

The data that support the findings of this study are available from the corresponding author upon reasonable request.

\section{Keywords}

biofabrication, extracellular matrix, immunomodulation, macrophages, melt electrofibrillation, melt electrowriting

Received: February 12, 2021

Revised: April 19, 2021

Published online: July 8, 2021

[1] J. Y. Huang, Y. W. Chen, C. Q. Tang, Y. Fei, H. Y. Wu, D. F. Ruan, M. E. Paul, X. Chen, Z. Yin, B. C. Heng, W. S. Chen, W. L. Shen, Cell. Mol. Life Sci. 2019, 76, 505.

[2] J. Kopf, S. Ulasevich, O. Baidukova, Y. Zhukova, J. W. C. Dunlop, P. Fratzl, P. Rikeit, P. Knaus, S. K. Poznyak, D. V. Andreeva, E. V. Skorb, Adv. Eng. Mater. 2016, 18, 476.

[3] B. J. Green, M. Panagiotakopoulou, F. M. Pramotton, G. Stefopoulos, S. O. Kelley, D. Poulikakos, A. Ferrari, Nano Lett. 2018, 18, 2140.

[4] A. K. Gaharwar, I. Singh, A. Khademhosseini, Nat. Rev. Mater. 2020, 5,686 .
[5] a) D. Grafahrend, K. H. Heffels, M. V. Beer, P. Gasteier, M. Moller, G. Boehm, P. D. Dalton, J. Groll, Nat. Mater. 2011, 10, 67; b) S. R. Caliari, J. A. Burdick, Nat. Methods 2016, 13, 405.

[6] M. J. Dalby, N. Gadegaard, R. O. C. Oreffo, Nat. Mater. 2014, 13, 558.

[7] S. Lenhert, M. B. Meier, U. Meyer, L. Chi, H. P. Wiesmann, Biomaterials 2005, 26, 563.

[8] a) M. Laranjeira, R. M. A. Domingues, R. Costa-Almeida, R. L. Reis, M. E. Gomes, Small 2017, 13, 1700689; b) M. J. Dalby, N. Gadegaard, R. Tare, A. Andar, M. O. Riehle, P. Herzyk, C. D. W. Wilkinson, R. O. C. Oreffo, Nat. Mater. 2007, 6, 997.

[9] S. Franz, S. Rammelt, D. Scharnweber, J. C. Simon, Biomaterials 2011, 32, 6692.

[10] J. Xue, S. V. Schmidt, J. Sander, A. Draffehn, W. Krebs, I. Quester, D. De Nardo, T. D. Gohel, M. Emde, L. Schmidleithner, H. Ganesan, A. Nino-Castro, M. R. Mallmann, L. Labzin, H. Theis, M. Kraut, M. Beyer, E. Latz, T. C. Freeman, T. Ulas, J. L. Schultze, Immunity 2014, 40, 274.

[11] a) F. O. Martinez, S. Gordon, F1000Prime Rep. 2014, 6, 13; b) R. Sridharan, A. R. Cameron, D. J. Kelly, C. J. Kearney, F. J. O'Brien, Mater. Today 2015, 18, 313.

[12] a) F. Y. McWhorter, C. T. Davis, W. F. Liu, Cell. Mol. Life Sci. 2015, 72, 1303; b) F. Y. McWhorter, T. T. Wang, P. Nguyen, T. Chung, W. F. Liu, Proc. Natl. Acad. Sci. USA 2013, 110, 17253.

[13] a) J. D. Bryers, C. M. Giachelli, B. D. Ratner, Biotechnol. Bioeng. 2012, 109, 1898; b) E. M. Sussman, M. C. Halpin, J. Muster, R. T. Moon, B. D. Ratner, Ann. Biomed. Eng. 2014, 42, 1508.

[14] T. Tylek, C. Blum, A. Hrynevich, K. Schlegelmilch, T. Schilling, P. D. Dalton, J. Groll, Biofabrication 2020, 12, 025007.

[15] T. Roszer, Mediators Inflammation 2015, 2015, 816460.

[16] a) V. S. Meli, P. K. Veerasubramanian, H. Atcha, Z. Reitz, T. L. Downing, W. F. Liu, J. Leukocyte Biol. 2019, 106, 283; b) V. F. Fiore, M. Krajnc, F. G. Quiroz, J. Levorse, H. A. Pasolli, S. Y. Shvartsman, E. Fuchs, Nature 2020, 585, 433.

[17] C. Q. Lin, M. J. Bissell, FASEB J. 1993, 7, 737.

[18] S. Chattopadhyay, R. T. Raines, Biopolymers 2014, 101, 821.

[19] T. D. Brown, F. Edin, N. Detta, A. D. Skelton, D. W. Hutmacher, P. D. Dalton, Mater. Sci. Eng., C 2014, 45, 698.

[20] T. Lorson, M. M. Lubtow, E. Wegener, M. S. Haider, S. Borova, D. Nahm, R. Jordan, M. Sokolski-Papkov, A. V. Kabanov, R. Luxenhofer, Biomaterials 2018, 178, 204.

[21] R. W. Moreadith, T. X. Viegas, M. D. Bentley, J. M. Harris, Z. H. Fang, K. Yoon, B. Dizman, R. Weimer, B. P. Rae, X. L. Li, C. Rader, D. Standaert, W. Olanow, Eur. Polym. J. 2017, 88, 524.

[22] S. Huber, R. Jordan, Colloid Polym. Sci. 2008, 286, 395.

[23] R. W. Moreadith, T. X. Viegas, M. D. Bentley, J. M. Harris, Z. Fang, K. Yoon, B. Dizman, R. Weimer, B. P. Rae, X. Li, C. Rader, D. Standaert, W. Olanow, Eur. Polym. J. 2017, 88, 524.

[24] G. Hochleitner, J. F. Hummer, R. Luxenhofer, J. Groll, Polymer 2014, 55, 5017.

[25] G. Sivalingam, R. Karthik, G. Madras, Polym. Degrad. Stab. 2004, $84,345$.

[26] G. Casella, L. Garzetti, A. T. Gatta, A. Finardi, C. Maiorino, F. Ruffini, G. Martino, L. Muzio, R. Furlan, J. Neuroinflammation 2016, 13, 139.

[27] P. Olczyk, L. Mencner, K. Komosinska-Vassev, Biomed Res. Int. 2014, 2014, 747584.

[28] T. M. Robinson, D. W. Hutmacher, P. D. Dalton, Adv. Funct. Mater. 2019, 29.

[29] a) R. B. Svensson, A. Herchenhan, T. Starborg, M. Larsen, K. E. Kadler, K. Qvortrup, S. P. Magnusson, Acta Biomater. 2017, 50 , 293; b) D. A. Parry, A. S. Craig, Biopolymers 1978, 17, 843.

[30] N. Jain, V. Vogel, Nat. Mater. 2018, 17, 1134.

[31] K. Garg, N. A. Pullen, C. A. Oskeritzian, J. J. Ryan, G. L. Bowlin, Biomaterials 2013, 34, 4439. 
[32] a) M. Bartneck, K. H. Heffels, Y. Pan, M. Bovi, G. ZwadloKlarwasser, J. Groll, Biomaterials 2012, 33, 4136; b) N. E. Paul, C. Skazik, M. Harwardt, M. Bartneck, B. Denecke, D. Klee, J. Salber, G. Zwadlo-Klarwasser, Biomaterials 2008, 29, 4056.

[33] M. J. Vassey, G. P. Figueredo, D. J. Scurr, A. S. Vasilevich, S. Vermeulen, A. Carlier, J. Luckett, N. R. M. Beijer, P. Williams, D. A. Winkler, J. de Boer, A. M. Ghaemmaghami, M. R. Alexander, Adv. Sci. 2020, 7, 1903392.

[34] H. M. Rostam, L. E. Fisher, A. L. Hook, L. Burroughs, J. C. Luckett, G. P. Figueredo, C. Mbadugha, A. C. K. Teo, A. Latif, L. Kammerling, M. Day, K. Lawler, D. Barrett, S. Elsheikh, M. Ilyas, D. A. Winkler, M. R. Alexander, A. M. Ghaemmaghami, Matter 2020, 2, 1564.

[35] E. Saino, M. L. Focarete, C. Gualandi, E. Emanuele, A. I. Cornaglia, M. Imbriani, L. Visai, Biomacromolecules 2011, 12, 1900.

[36] F. J. O'Brien, Mater. Today 2011, 14, 88.

[37] J. Jiang, Z. Li, H. Wang, Y. Wang, M. A. Carlson, M. J. Teusink, M. R. MacEwan, L. Gu, J. Xie, Adv. Healthcare Mater. 2016, 5, 2993.

[38] R. M. Visalakshan, M. N. MacGregor, S. Sasidharan, A. Ghazaryan, A. M. Mierczynska-Vasilev, S. Morsbach, V. Mailander, K. Landfester, J. D. Hayball, K. Vasilev, ACS Appl. Mater. Interfaces 2019, 11, 27615.

[39] T. Tylek, T. Schilling, K. Schlegelmilch, M. Ries, M. Rudert, F. Jakob, J. Groll, Sci. Rep. 2019, 9, 3533.

[40] a) A. Toniolo, G. P. Fadini, S. Tedesco, R. Cappellari, E. Vegeto, A. Maggi, A. Avogaro, C. Bolego, A. Cignarella, J. Clin. Endo- crinol. Metab. 2015, 100, E50; b) Y. Yang, N. S. Luo, R. Ying, Y. Xie, J. Y. Chen, X. Q. Wang, Z. J. Gu, J. T. Mai, W. H. Liu, M. X. Wu, Z. T. Chen, Y. B. Fang, H. F. Zhang, Z. Y. Zuo, J. F. Wang, Y. X. Chen, Int. J. Mol. Med. 2017, 40, 558.

[41] A. Etzerodt, S. K. Moestrup, Antioxid. Redox Signaling 2013, 18, 2352.

[42] a) R. J. Snyder, J. Lantis, R. S. Kirsner, V. Shah, M. Molyneaux, M. J. Carter, Wound Repair Regener. 2016, 24, 613; b) L. Claes, S. Recknagel, A. Ignatius, Nat. Rev. Rheumatol. 2012, 8, 133.

[43] P. L. Graney, S. Ben-Shaul, S. Landau, A. Bajpai, B. Singh, J. Eager, A. Cohen, S. Levenberg, K. L. Spiller, Sci. Adv. 2020, 6, eaay6391.

[44] E. M. O'Brien, G. E. Risser, K. L. Spiller, Adv. Drug Delivery Rev. 2019, 149-150, 85.

[45] A. E. Koch, P. J. Polverini, S. L. Kunkel, L. A. Harlow, L. A. Dipietro, V. M. Elner, S. G. Elner, R. M. Strieter, Science 1992, 258, 1798.

[46] L. A. Wells, M. S. Valic, A. Lisovsky, M. V. Sefton, Isr. J. Chem. 2013, $53,637$.

[47] a) A. G. Stewart, B. Thomas, J. Koff, Respirology 2018, 23, 1096; b) C. E. Witherel, D. Abebayehu, T. H. Barker, K. L. Spiller, Adv. Healthcare Mater. 2019, 8, 1801451; c) J. Sapudom, W. K. E. Mohamed, A. Garcia-Sabate, A. Alatoom, S. Karaman, N. Mahtani, J. C. Teo, Bioengineering 2020, 7, 1.

[48] G. Hochleitner, T. Jungst, T. D. Brown, K. Hahn, C. Moseke, F. Jakob, P. D. Dalton, J. Groll, Biofabrication 2015, 7, 035002. 